\title{
Accelerating Renewable Connections through Coupling Demand and Distributed Generation
}

\author{
Milana Plecas, Simon Gill and Ivana Kockar \\ Department of Electronic and Electrical Engineering \\ University of Strathclyde \\ Glasgow, UK \\ milana.plecas; simon.gill; ivana.kockar \{@strath.ac.uk\}
}

\begin{abstract}
The objective of this paper is to investigate the options for using local demand to accelerate the connection of renewable Distributed Generation (DG) capacity. It presents a range of architectures for operating Distributed Energy Systems (DESs) that contain local demand and distributed generation. The concept of a DES is that demand is supplied by local DG either using privately owned distribution assets or a public distribution network owned by a Distribution Network Operator (DNO). Operation of a DES can help manage variability in DG output, reduce curtailment in Active Network Management (ANM) schemes, and assist the DNO in managing network constraints. They also provide a move towards local trading of electricity with potential financial and non-financial benefits to both distributed generators and local demand customers.
\end{abstract}

Keywords- Distributed Generation; Distributed Energy Systems; Distribution Network Operator; Flexible Demand

\section{INTRODUCTION}

The integration of distributed generation (DG) connected at lower voltage levels introduces significant changes in power system operation and planning procedures, as well as in the design of electricity markets. Traditionally passive distribution networks, designed around centralized power generation with the unique direction of electricity flow from the transmission system to the consumers, have become active systems with bidirectional power flows. This has led to certain network management issues related to voltage rise and line capacity, which means that in order to facilitate more DG, there is a need for Distribution Network Operators (DNOs) to more actively operate their networks. Allowing further DG connections to the distribution networks that were considered full under existing management strategies, will imply some limitations on the new generator outputs.

These limitations can be overcome either by using export limited connections or Active Network Management (ANM). The former can be used in instances where the connection capacity is lower than the maximum output of the generator. It places a fixed limit on what may be exported into the network, but with a possibility of additional generation output if it is consumed locally. ANM has been developed over the past decade in the UK instead of costly and time consuming network reinforcements [1]. It extends traditional limits on DG capacity of the existing network by the real time management of generation and loads on the network to match the available network capacity [1]. ANM monitors the real time network capacity and manages connections in accordance with their commercial arrangements and Principles of Access (PoA) [1].

The most commonly used PoA in the UK is the Last in First off (LIFO), in which the last connected generator is restricted before the first. However other methods have also been investigated [2]. There are two types of commercial arrangements within an ANM scheme, (i) 'firm connection' which allows generator to export power to the network at all times and (ii) 'non-firm connection' which does not guaranteed access to the network at all times but rather stipulates that the generator would need to curtail its output whenever instructed to do so by a DNO to avoid network limits being exceeded.

In the case of variable renewable generators such as wind, solar and run-of-river-hydro, both non-firm and export limited connections will lead to the loss of opportunity to generate electricity from free, clean renewable sources. Therefore, the connection of a local flexible demand presents an opportunity to use this otherwise curtailed generation.

In the UK, Ofgem, which acts as a regulator, has recognized a need to entice DNOs to look into implementations of new innovative solutions and/or technologies to achieve low carbon networks with increased penetration of DGs. To that end, a number of Low Carbon Network Fund (LCNF) projects have been co-funded by Ofgem and industry to address these issues [3], such as The Northern Isles New Energy Solutions (NINES) led by SSE [4], Low Carbon Hub led by Western Power Distribution [5], and Accelerating Renewable Connections (ARC) led by SP Energy Networks [6]. The ARC project investigates alternative methods to allow integration of new DG connections onto a distribution network that previously was believed to be at full capacity.

The concept of a Distributed Energy System (DES) is that demand is supplied by a local DG either using privately owned distribution assets or a public distribution network owned by a DNO. The net power represents a DES's contribution to any network constraints, and as such, there is a potential for the system to receive connection capacities based on the net power rather than generated power. Although, the concept of reducing curtailment through the action of local demand is intuitively simple, the practicality of implementing 
such a scheme can be complex. Firstly, any scheme needs to maintain the technical integrity of the electrical system including fail-safe mechanisms that satisfy the DNO's operational requirements. In addition, the commercial arrangements between generator and demand must satisfy electricity regulation and maintain the liberalized market principles of competition in the wholesale and supply markets. Furthermore, generation, supply, and distribution activities are usually licensed activities and, in the UK any scheme undertaking any of these must either fulfil licences requirements or qualify for exemptions [7]. Finally, if operating as part of an ANM scheme, a local energy project must be capable of integrating with the communications, control systems, and wider principles of access imposed by ANM. Thus, here are significant challenges facing both DNO and community energy schemes that aim to couple local flexible demand with local generation.

The objective of this paper is to highlight benefits and challenges of creating a DES as well as to lay out different DES architectures. The DES architectures discussed here range from simple behind-the-meter options (which are already implemented, or can be easily implemented in the UK by using or extending ANM schemes that are becoming Business-as-Usual approaches for a number of UK DNOs) to emerging, more complex, demand aggregation and local market schemes:

- Private wire systems: The connection of DG and demand over private owned distribution assets and a single point-of-connection between the scheme and the DNO owned network.

- Virtual private wire systems: A link between generators and either one, or a small number of flexible demands through the DNO owned network.

- Demand aggregation: Flexibility is achieved by responses from a large number of small demands, such as domestic properties. This flexibility is aggregated to provide a significant overall affect. The aggregator itself acts as a point of contact for the ANM scheme and associated generators and manages participation of flexible demand so to achieve requested flexibility at times when needed.

- Local markets: Demand and DG engage in a local market to either increase demand or decrease generation in such a way as to avoid constraints.

\section{Distributed ENERGy Systems: BENEFITS AND CHALLENGIES}

There is the potential for both demand customers and generators to benefit from coupling together to form a DES. Benefits can take the form of financial incentives with increased payments to generators and decreased cost of energy for consumers. There are also significant reputational benefits, for example through the traceability of electricity that such a scheme can provide. DES schemes can reduce congestion further up the distribution network and there may be a benefit to the DES in terms of reduction of Use of System Charges for demand and generation, and to the DNO in terms of infrastructure investment deferral. Apart from above benefits, there are also operational and regulatory difficulties associated with setting up such schemes.

The exact nature of the benefits and challenges available to demand customers and generators will depend on the type of connection agreement available to the generator. Until recently only firm connections agreements have been available in the $\mathrm{UK}$, and therefore most distributed generators operate under a firm connection agreements giving them network access at all times up to their rated capacity; such an arrangement is considered business as usual. Another agreements being considered by DNOs are an export limited connection and nonfirm connections agreements introduced by the development of ANM schemes. These are explained below:

- Firm connection: This is the standard connection offer in which the generator receives a firm connection equal to the rated capacity of the generator.

- Export limited connection: If there is limited capacity for DG to connect to the local distribution network, a generator can be offered a firm connection for part of the proposed DG capacity. Accepting such an export limited connection will result in the generator being de-rated to the capacity of the connection offer. This reduces potential income, however investors may accept such an offer if the economics of the scheme remain viable, or if there is the potential for greater network capacity to be released in the future.

- ANM non-firm connection: A non-firm ANM connection offer provides network capacity to a generator up to its installed capacity according to a defined PoA. At any time, the maximum generator output can be limited to any point between the zero and maximum capacity, and the generator must respond to ANM curtailment signals by reducing generation to below the specified level. In existing ANM schemes, non-firm connection offers include a non-binding estimate of likely curtailment levels. These estimates are typically calculated using historical data. If only minor curtailment is predicted, the generator will expect to generate at full capacity the majority of the time and the project is likely to be financially viable.

\section{A. Benefits of DES}

For each of the network connection types there are benefits associated with coupling demand to generators in a DES. In each case generators and demand customers will compare the benefits associated with participating in a DES with those associated with standard arrangements; only where the benefits of a DES are greater will a scheme be viable. For generators with firm connection agreements, a DES will need to provide benefits which the generator feels are more valuable than those which can be realized under a standard power purchase agreement. For example, if a generator can get a power purchase agreement that pays $£ 50 / \mathrm{MWh}$ the DES arrangement must either provide a higher price per MWh, or other benefits such as reduced risk or reputational benefits associated with supplying local demand. For generators experiencing curtailment, either under export limited connection or ANM, 
curtailed generation represents a loss of revenue. Therefore, the ability of a DES to reduce curtailment allows the generator to derive some benefit by potentially reducing the curtailment for a significant period of operation.

Table I gives a summary of the benefits associated with demand and DG in a DES, as well as the specific benefits relating to generators. Each type of connection agreement is discussed below.

\section{1) Benefits of a DES associated with firm connected DG}

With a firm network connection, the generator does not have the risk of curtailment. All of its generation can be sold under standard agreements with a supplier, usually through a Power Purchase Agreement (PPA). For small generators, the price per unit in the PPA will usually be set by the relevant Feed-In Tariff (FIT) rate, while for larger generators income will also be generated by the sale of Renewable Obligation Certificate (ROC). In this situation, although a generator with firm connection does not face curtailment, it can still benefit from taking part in a DES if it can negotiate a higher price for its power output.

The benefits for demand customers participating in a DES include non-monetary benefits related to the local, renewable and traceable nature of the electricity used. Domestic and business customers may consider buying electricity guaranteed to be from locally owned generation even if the prices are similar or slightly higher than those on the open market as it can provide a guarantee that their payment goes directly to a local renewable generator.

\section{2) Benefits of a DES associated with an export limited connected $D G$}

With an export limited connection agreement, the technical ability of a generator to produce electricity beyond the connection limit is of zero value. To access any benefit from the capped generator capacity, a DG needs to find a way of using that additional generation to supply local demand that is behind the network constraint that is causing the limited connection.

For wind, solar, and run-of-river hydro generators, the short-run cost of generation is close to zero. Therefore the ability to receive even a small revenue from generation beyond its export limited capacity represents a net benefit. By comparison, demand customers under normal arrangements pay the market rate for supply of electricity. Therefore a DES which allows export-limited generators to be paid even a low price for generation beyond the export limit and allows customers to pay less than market rate can provide greater benefit to both generator and demand customers when compared to standard arrangements.

Whilst there is obvious benefit in the use of a DES to increase generation beyond the export-limit, all generation up to the export limit can be sold under a standard PPA and as in the case of a firm connection agreement. The quantity and timing of additional generation that would otherwise be curtailed depends on the generator type and will have an impact on the frequency with which these benefits can be realized. For a run-of-river hydro scheme with a good resource, there is the potential to generate at full capacity for a significant proportion of the year. For a wind generator, the availability of excess generation will depend on the available wind resource. Therefore the design of a DES scheme will need to take into account the likely availability of otherwise curtailed generation. As an example there is a question over whether demand customers buy all their electricity through the DES or retain existing supply arrangements for the majority of their supply with the DES acting as a 'top up'. These decisions depend in part on the likely distribution across the year of generation that would otherwise be curtailed.

\section{3) Benefits of DES associated with ANM managed DG}

The benefits for non-firm generators in an ANM scheme of making use of otherwise curtailed generation are similar to those associated with export limited connections. Under existing ANM arrangements, curtailment instruction must be observed by the reduction of generation. Curtailed generation therefore has zero value. As with export limited connections, both DG and demand will benefit if a DES can arrange for that otherwise curtailed generation to be used, and if they can agree a price which is less than a market rate for supply of electricity.

With an ANM connection, the timing of curtailment is less certain and more likely to show significant seasonal variations when compared to that of an export limited connection. For example if base-load demand is significantly higher in winter than summer, curtailment under ANM may occur almost solely during summer.

TABLE I. A SUMMARY OF THE BENEFITS ACCRUING THE GENERATORS AND DEMAND CONSUMERS UNDER A DES

\begin{tabular}{|c|c|c|}
\hline $\begin{array}{c}\text { Connection } \\
\text { type }\end{array}$ & DES advantage to generator & DES advantage to demand \\
\hline $\begin{array}{c}\text { Firm } \\
\text { connection }\end{array}$ & $\begin{array}{l}\text { Potentially increased price per } \\
\text { unit of generation compared } \\
\text { to available PPAs. }\end{array}$ & $\begin{array}{l}\text { - Reputational benefit from } \\
\text { traceable, local and } \\
\text { renewable electricity. }\end{array}$ \\
\hline $\begin{array}{l}\text { Export } \\
\text { limited } \\
\text { connection }\end{array}$ & $\begin{array}{l}\text { - Ability to increase generation } \\
\text { beyond export limit. } \\
\text { - Potentially increased price per } \\
\text { unit of generation compared } \\
\text { to available PPAs. }\end{array}$ & $\begin{array}{l}\text { - Reduced costs of } \\
\text { electricity. } \\
\text { - Reputational benefit from } \\
\text { traceable, local and } \\
\text { renewable electricity. }\end{array}$ \\
\hline $\begin{array}{l}\text { ANM } \\
\text { non-firm } \\
\text { connection }\end{array}$ & $\begin{array}{l}\text { - Ability to generate beyond the } \\
\text { current ANM calculated limit. } \\
\text { - Potentially increased price per } \\
\text { unit of generation compared } \\
\text { to available PPAs. }\end{array}$ & $\begin{array}{l}\text { - Reduced costs of electricity } \\
\text { during curtailment events. } \\
\text { - Reputational benefit from } \\
\text { traceable, local and } \\
\text { renewable electricity. }\end{array}$ \\
\hline
\end{tabular}

In the discussion above it is assumed that the short run cost of generation is close to zero and once curtailed the ability to generate from that resource is lost. This is true for many renewable technologies such as wind where there are no fuel costs and the wind resource cannot be stored. For non-variable renewable generators such as Anaerobic Digesters these assumptions are not necessarily true. Anaerobic Digesters burn fuel which can be stored and reducing generation for a curtailment event means the fuel can be stored and used later. The economics for these cases therefore depend on the relative availability of fuel compared to the capacity of the generator. If there is an excess of fuel, curtailment means losing the opportunity to use fuel; whereas if there is a scarcity of fuel, curtailment does not mean the loss of opportunity to generate, as fuel could be stored and used later.

Synthesizing these benefits into a workable commercial model is a significant challenge for any DES scheme and will 
involve negotiations involving generators, demand customers and suppliers. Although the DNO is not involved in setting up this model, distribution networks are important in facilitating DESs, and DNOs must ensure that any scheme maintains the integrity and safety of its network.

\section{B. Challenges of DES}

Several areas in which setting up a DES may create challenges are identified below.

\section{1) Operational challenges}

Combining demand and DG requires that the scheme is capable of controlling the net output as opposed to the generated output. This creates additional complexity as both additional measurements and control logic are needed. Operational requirements include the need for secure fail-safe systems to ensure that the network remains safe and secure during fault conditions. When operating within an ANM scheme, the DES must meet ANM requirements such as response time-scales.

\section{2) Electrical connection arrangements}

The electrical link between demand and DG can take several forms depending on their geographical location and the relative number of sites involved. Demand and DG within a DES can be electrically linked across the DNO owned distribution network or via privately owned infrastructure with a single point-of-connection to the distribution network. In both cases, the contribution to any network constraints must be considered, including the roll of electrical losses.

\section{3) Communication challenge}

Communications within a DES and with other systems such as an ANM system are required for secure operation. ANM schemes have the potential to interface with demand as well as distributed generators, although with a larger number of demands (for example if using domestic demand at multiple houses) it is likely that an aggregator will be required to provide a single point of contact between the DNO or an ANM scheme and the flexible demand.

\section{4) Commercial arrangement}

The commercial arrangements needed to run a DES are themselves novel. The model chosen depends on whether flexible demand is located on the same site as the generator, and on whether the scheme is connected using a private network or the public distribution network. The objective of a DES is to provide a method of increasing the return for generators and demand customers compared to standard arrangements. In all cases, the arrangements must satisfy the regulatory requirements, however, it is also important that the DNOs provide guidance on the feasibility of the connection.

\section{5) Regulatory issues}

Distribution and supply of electricity are all licensed activities and meeting the full licence conditions is likely to be well beyond the means of a community project. A number of options exist for smaller schemes to reduce the financial and risk related burdens that licence conditions place on participants. Alternative methods of meeting regulatory requirements are possible, for example linking the schemes to larger supply companies, which can provide services as licensed providers.

\section{6) Principles of access}

A clear PoA is needed under any scheme to ensure clear simple and fair distribution of curtailment among multiple generators, and the inclusion of DESs and the roll of demand in managing curtailment must be considered. Existing ANM principles of access based on LIFO are clear and simple and allow relatively easy estimation of likely curtailment. However, these estimates often make use of the expected demand levels on the distribution network when determining the "network capacity'. The move towards flexible demand has the potential to disrupt these assumptions and calculations. Schemes developed in conjunction with one particular generator can therefore affect curtailment applied to other generators.

\section{FOUR DES ARCHITECTURES}

DESs must be technically feasible, operate securely with robust fail-safe mechanisms, and satisfy the regulatory and commercial environments of the UK electricity sector. There are a wide range of architectures, which can achieve these objectives depending on the type of demand, locations of generators, whether curtailment is an issue, and the operation of ANM if linked to the scheme. This section presents four such architectures. The architectures have been chosen to offer options, which are feasible for ANM, and non-ANM connected generators, existing or new electrical demand, and flexible or inflexible demand. Note that due to limited space commercial aspects have not been discussed here.

\section{A. Private wire systems}

A private wire $(\mathrm{PW})$ system is a localized private electricity network that directly connects demand and generation. It can be seen as a microgrid that supplies domestic customers and businesses. Its size can range from single sites to urban area or housing development. A DG is located either on the same site as the demand or a geographically close site. The DG and demand are directly connected via the private wire network and the entire PW network is connected to the distribution network via a single point-of-connection.

The DG capacity within a PW network can be larger or smaller than demand with the difference between the two being imported or exported through the point-of-connection with the distribution network. The export capacity of a PW system can potentially receive any of the connections available to standalone generators: firm connection, export limited, or a non-firm connection under an ANM scheme. Under export limited and ANM connections, a local controller is used to control DG and demand on the PW network and make sure that the PW system export capacity not exceeds the distribution network constraints.

A schematic diagram of a PW system operating within an ANM scheme is shown in Fig. 1. It shows the ANM scheme linked to the local controller of a PW system. The ANM scheme monitors the power flow through the export meter. If the PW system has an export limited connection or a non-firm ANM connection, a controller is required to manage export. For ANM it must adjust the power injection of the PW system within the set-point limits defined by the ANM scheme. For 
example, if there is surplus power, the PW controller must either curtail the generation or increase flexible demand.

Since the PW system is independent of the distribution network operator, distribution use of system charges will be reduced, as there is a reduction of import. In some cases, if demand receives a supply from both the private wire and the distribution network, customers may have a switch them to manually revert to the distribution network.

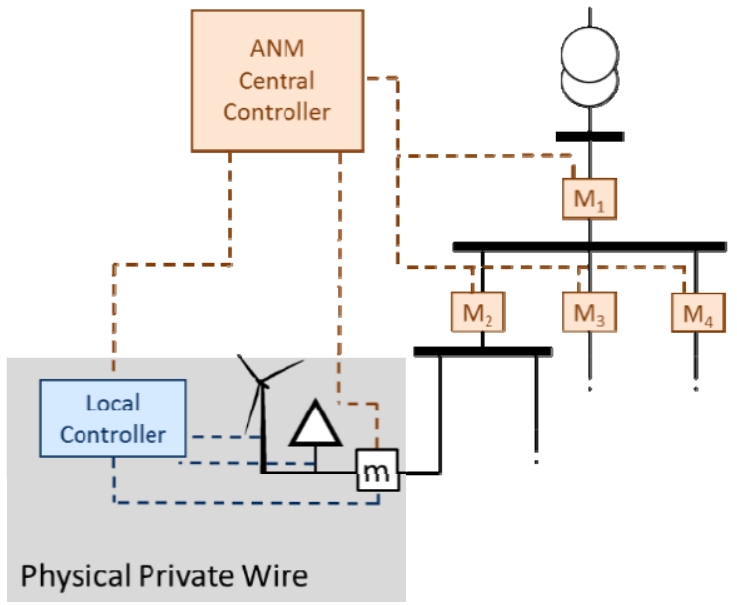

Fig. 1. Schematic of a Private Wire System within an ANM scheme

There are a couple of PW systems trialled in the UK. A hydro-electric generation scheme on Applecross in the northwest Highlands connects a run-of-river scheme, with an installed capacity of $90 \mathrm{~kW}$, and a district heating scheme in order to use $40 \mathrm{~kW}$ which would be curtailed as the maximum grid connection currently available is $50 \mathrm{~kW}$ [8]. The village of Findhorn, located in the north of Scotland, is served by a privately owned distribution network connecting domestic and small commercial properties as well as a small wind farm. Flow into and out of Findhorn is monitored as the interface with the public distribution network [9].

\section{B. Virtual private wire systems}

A virtual private wire (VPW) system consists of demand and generation connected at different points to the distribution network and power flowing between them across the distribution network. The VPW can be characterised by its net contribution to a distribution constraint in a similar way to a PW. The maximum export of a VPW can be limited at a level below the installed DG capacity or managed through an ANM scheme, in the same way as a PW system. The difference between the two architectures is that power flows between demand and generation across the distribution network rather than through a private network. The section of distribution network involved in these internal VPW flows is below any network constraint locations. In an ANM scheme, a VPW network can interact as a single entity with the ability to interpret a single ANM set point by either reducing generation or increasing demand to produce the same aggregate result.

A VPW makes use of the existing power system infrastructure avoiding duplication. The important aspects of such a scheme are the monitoring and control components. Fig. 2 shows a schematic for a VPW operating within a subsection of a distribution network with ANM. The demand is located outside the wind farm meter, although the communications and control architecture are almost identical to that shown in Fig. 1. The ANM scheme is linked to the controller of a VPW and monitors power flow through the meters of generation and demand.

The VPW system connects DG and demand via the existing distribution network, and is to be seen by the wider distribution network and an ANM scheme as a single entity with a single power injection. The DNO will stipulate the point at which the virtual net injection of power are calculated for, this may be the relevant constraint location, or the point at which power flows from and to the generator and demand converge.

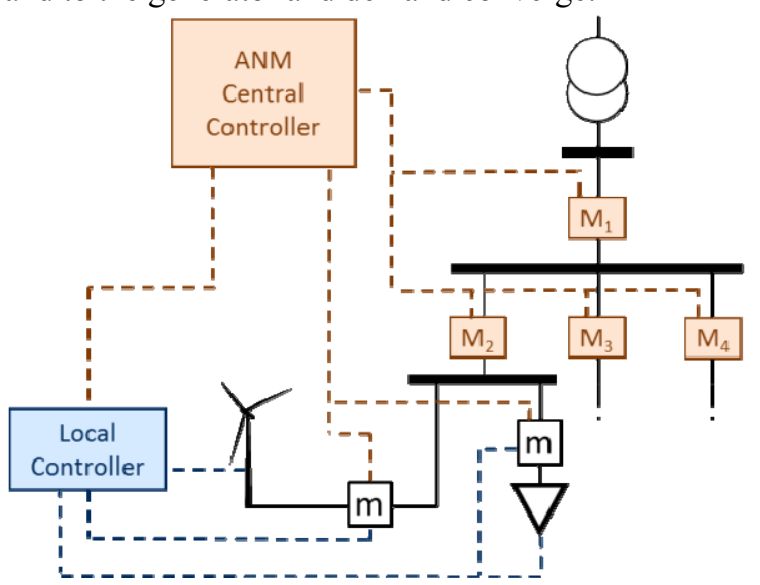

Fig. 2. Schematic of a Virtual Private Wire System within an ANM scheme

In the case of an export limited connection, the net export of the VPW is limited below the capacity of generation. A control system similar to that for a PW system must be in place to increase demand or curtail generation if export approaches the limit. This controller monitors generation and demand on the VPW and ensures that the combined virtual injection is maintained within limits. In the case of ANM the VPW controller can report the aggregate power injection to the ANM scheme, or the ANM scheme may choose to monitor each of the VPW assets individually and carry out its own aggregation calculation.

The inclusion of a VPW in an ANM scheme raises a number of technical challenges for the ANM operator and the design of the VPW. These relate to the interpretation of ANM signals and the required response time for curtailment. The provision of set-points by the ANM scheme to a VPW can be achieved in the same way as for non-firm generators: a single set-point is sent and interpreted as relating to net power injection rather than generator output. In the event that the VPW fails to comply with a set point within the specified period, the ANM scheme will wish to trip the generator assets of the VPW; demand assets should be left connected. This requires a direct communication link between the ANM scheme and the breakers at the DG assets. The ANM scheme will monitor the net power injection of the VPW.

ANM actions are triggered at particular values of power system variables (power flow or voltage) set below the absolute limit. One consideration for demand flexibility within an ANM scheme is the timescale of response. Generators are 
given a particular time to respond to a trim set point within ANM. If a VPW (or a PW) intends to use demand response to decrease net export, the time-scale of demand response must be considered.

VPW systems are rare in the UK with DNOs general unwilling to facilitate them. One DES set up by Woking Council links renewable and CHP generation with demand in council premises, local commercial buildings, and social housing [10]. The scheme involves a number of components including private electrical wires, private district heating network and some provision of electricity to social housing around the town via the public distribution network. This final component forms a VPW system and makes use of the local distribution network owned and operated by UK Power Distribution.

\section{Demand aggregation}

Domestic consumers and small businesses can in aggregation provide a significant level of demand flexibility. Accessing this demand means communicating and controlling a large number of small demands in a coordinated way. A DNO or an ANM scheme operator is unlikely to have the capacity to communicate directly with each demand, and generators will not want to make separate arrangements with a large number of households. In addition, variations in individual loads are often difficult to predict whereas useful flexibility emerges from the statistic effect of aggregated demand.

In schemes involving a local supply company, the supplier may acts as an aggregator if it supplies to a large number of small loads. A non-firm generator informs the local supply company of a curtailment event, and the supply company creates an increase in demand, which reduces the curtailment.

However, there may be situations in which demand is not aligned with one particular generator. There are a number of options for such schemes. The DNO or ANM can consider incorporating demand within an ANM scheme, or the DNO may instigate a scheme, which facilitates supply companies setting up 'flexible tariffs' based on information provided by ANM. A second option is that demand makes its flexibility available to multiple generators. For example, in an ANM scheme under existing LIFO principles of access, flexible demand could offer its flexibility to whichever generator is currently the marginal generator.

The management of multiple small demands in either of these situations can be organized by a demand aggregation. The aggregator acts as a single point-of-contact for other actors within the distribution environment: ANM operators, DNOs, and generators.

A demand aggregation system makes use of the existing electrical network to supply electricity to the consumers, in the same way as is done at present. The innovation of an aggregation scheme is to allow the manipulation of many existing (and new) electrical demands through the current electrical infrastructure. Aggregation requires the roll out of smart meters to all demand customers to provide an accurate record of the timing at which demand is taken and allow the calculation of the flexibility provided by each individual load.

With smart meters installed for all customers, the basic communication and control actions of the aggregator are shown in Fig. 3. The aggregator monitors the current demand of each individual customer and aggregates this to give a total demand. It receives a set-point demand level from an external system; this can be a generator or the ANM system for example. The aggregator uses some control algorithm to calculate how to meet the set point and send control signals out to individual customer's premises.

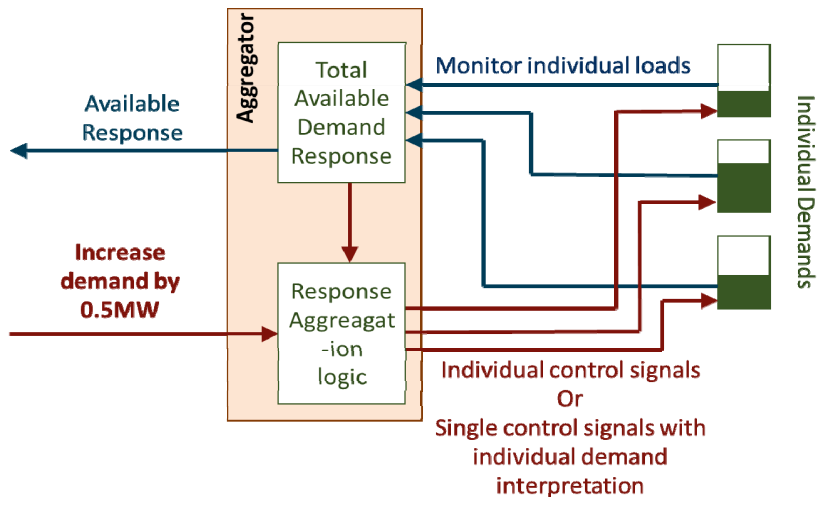

Fig. 3. Schematic representation of an aggregator

Demand aggregation to assist in the management of a particular constraint must be behind that constraint.

One of the projects where demand aggregation has been trialed is the Northern Isles New Energy Solutions Project (NINES) on Shetland. It uses demand aggregation to manage electric storage heaters across the islands power system [11].

\section{Local markets}

A local market is a mechanism in which both non-firm generators and flexible demand units take part via offers and bids in the buying and selling of electricity. It allows consumers to buy electricity from local producers and the local market operator to balance the system in order to satisfy both demand and generation needs whilst maintaining network constraints. The market price will be influenced by the weather, price of fuel and emission allowances. The local market can interact with an ANM scheme through market operator.

One example of a local market is a curtailment market: under this arrangement, the existing ANM principles of access are applied to calculate initial curtailment levels. Then curtailed generators and flexible demand submit offers and bids for increased generation and increased demand respectively. A local market operator then clears the market, and passes the reduced curtailment levels to the ANM scheme for implementation.

A schematic of a local curtailment market system operating with an ANM scheme is shown in Fig. 4. It shows the ANM scheme linked to the local controller that controls non-firm generators and demands.

The ANM scheme monitors power flow through the meters of generation and demands. Demands are spread across the 
distribution network and are not associated with a particular generator. They can be monitored individually via demand meters or aggregated via load aggregator that controls a group of demands. The market mechanism has a close-to-real-time price signal which acts as a balancing method for managing distribution congestion. There is a clearing period, after which the local market operator clears the market and sends results to the ANM scheme for implementation. A price signal close to real-time minimizes forecast errors caused by volatile outputs of renewable resources.

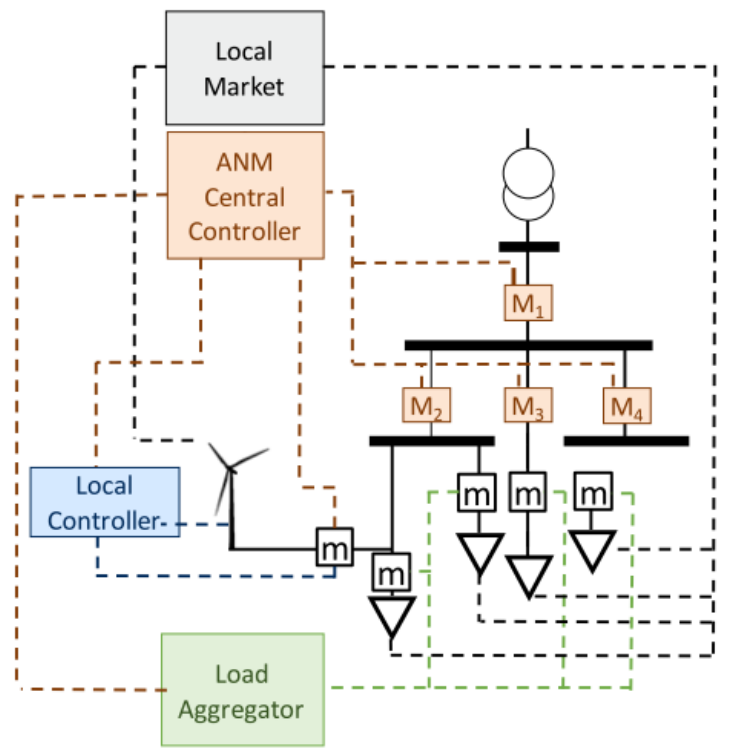

Fig. 4. Schematic of a Local Market System

The power transfer components of the network remain unchanged. The market operator requires communications infrastructure between itself and participants in the market. This allows participants to place bids and offers and the market operator to inform the results of market clearing. The control aspect of the market involves implementing the cleared market: sending and maintaining set points for generators and loads. This can be achieved through an ANM scheme.

Local markets at distribution level have been facilitated outside the UK. A price-responsive electricity market with 5minute clearing intervals for additional generation has been implemented on the Olympic Peninsula [12] and the bid-less EcoGrid EU real-time market concept on the Danish island Bornholm [13].

\section{CONCLUSIONS}

This paper presented an overview of the issues associated with coupling demand and DG to mitigate curtailment of renewable generators. It has discussed the benefits of such schemes for the generator and demand customers, and the regulatory difficulties associated with setting up such schemes. In addition, possible DES arrangement that allow renewable distributed generators with connection agreements lower than the installed generation capacity (export limited connections) as well as distributed generators connected with non-firm connection agreements, both managed under an ANM scheme, were presented The architectures range from simple behindthe-meter options to a complex local market scheme and the paper discussed several areas in which setting up a DES may create challenges.

\section{ACKNOWLEDGMENT}

The authors gratefully acknowledge useful discussions with Community Energy Scotland and support from SP Energy Networks.

\section{REFERENCES}

[1] Energy Networks Association. (2015, July). Active Network Management Good Practice Guide. [Online]. Available: http://www.energynetworks.org/assets/files/news/publications/1500205 ENA ANM report AW online.pdf.

[2] I. Kockar, E. Davidson, M. Dolan, and G. Ault, "Distributed generation Access and Power Flow Management", Presented at IEEE/PES General Meeting, Minneapolis, US, July 2010.

[3] Ofgem. (Last updated 2016, July). Low Carbon Networks Fund. [Online]. Available: https://www.ofgem.gov.uk/electricity/distributionnetworks/network-innovation/low-carbon-networks-fund.

[4] S. Gill, M. Dolan, A. Emhemed, I. Kockar, M. Barnacle, G. Ault, and C. Mathieson, "Increasing renewable penetration on islanded networks through active network management: a case study from Shetland," Renewable Power Generation, IET, vol. 9, no. 5, pp. 453-465, July 2015.

[5] Western Power Distribution. (2015, May). Low Carbon Hub project close down report. [Online]. Available: http://www.smarter networks.org/Files/Low_Carbon_Hub_151022125750.pdf.

[6] SP Energy Networks. (2012, Nov.). Accelerating Renewable Connections Full Submission. [Online]. Available: http://www.smarternetworks.org/Files/ARC_130115123252.pdf.

[7] Ofgem. (Last updated 2016, May). Licence Conditions. [Online]. Available: $\quad$ https://www.ofgem.gov.uk/licences-codes-andstandards/licences/licence-conditions

[8] Applecross Community Hydro project. [Online]. Available: http://www.applecrosshydro.scot/the-scheme/.

[9] Findhorn Ecovillage project. [Online]. Available: http://www.ecovillagefindhorn.com/.

[10] J. P. Thorp and L. Curran, "Affordable and Sustainable Energy in the Borough of Woking in the United Kingdom," Bulletin of Science, Technology \& Society, vol. 29, pp. 159-163, April 2009,

[11] M. J. Dolan, S. Gill, G. Ault; M. Barnacle, C. Foote, and G. Bell, "Modelling and delivery of an active network management scheme for the Northern Isles new energy solutions project," CIRED 2013, 22nd International Conference on Electricity Distribution, Stockholm, June 2013.

[12] D. J. Hammerstrom, "Pacific Northwest GridWiseTM Testbed Demonstration Projects," prepared for U.S. Department of Energy by the Pacific Northwest National Laboratory, Richland, WA, 2007.

[13] EcoGrid EU project. (2015). EcoGrid EU: From Implementation to Demonstration. [Online]. Available: http://www.euecogrid.net/images/Documents/150917_EcoGrid\%20EU\%20Implement ation $\% 20$ to $\% 20$ Demonstration.pdf. 